\title{
Some bounds for the $Z$-eigenpair of nonnegative tensors
}

\author{
Xiaoyu Ma' and Yisheng Song ${ }^{1 *}$ (1)
}

\author{
"Correspondence: \\ songyisheng1@gmail.com \\ 'School of Mathematics and \\ Information Science and Henan \\ Engineering Laboratory for Big Data \\ Statistical Analysis and Optimal \\ Control, Henan Normal University, \\ XinXiang, P.R. China
}

\begin{abstract}
Tensor eigenvalue problem is one of important research topics in tensor theory. In this manuscript, we consider the properties of $Z$-eigenpair of irreducible nonnegative tensors. By estimating the ratio of the smallest and largest components of a positive $Z$-eigenvector for a nonnegative tensor, we present some bounds for the eigenvector and Z-spectral radius of an irreducible and weakly symmetric nonnegative tensor. The proposed bounds complement and extend some existing results. Finally, several examples are given to show that such a bound is different from one given in the literature.
\end{abstract}

MSC: 90C26; 90C22; 65K10; 65F15; 15A72; 15A48; 15A69; 65K05; 90C30

Keywords: Irreducible nonnegative tensors; Z-eigenpair; Spectral radius; Weakly symmetric

\section{Introduction}

Matrix theory is one of the most fundamental tools of mathematics exploration and scientific research $[2,12]$. As a higher-order generalization of a matrix, tensors and their properties are widely used in a great variety of fields, such as gravitational theory and quantum mechanics in physics [32, 42], large-scale date analysis [18], hypergraph spectral theory [33, 43], social network data analytics [16, 48], automatical control [27], the best rank-one approximations in statistical data analysis [17, 49], complementarity problems $[1,7,9,10,15,24-26,37,38,40,41]$, etc. As a significant knowledge point of tensor theory, tensor eigenvalues is one of the most popular research topics in recent years, and gradually appears in many research and application fields.

In 2005, Qi [28] introduced the concept of eigenvalues for symmetric tensors. At the same time, this concept was simultaneously introduced by Lim [23], but he only considered the case when the eigenpairs are real. Since then, the tensor eigenvalue theory has attracted great attention and developed rapidly over the last decades. However, in order to find an eigenvalue or eigenvector of a higher-order tensor, it is necessary to solve a system of higher-degree polynomial equations with multiple variables [29, 31]. This means that it will be extremely difficult to solve the tensor eigenvalue problem when the order of such a tensor is very high. Therefore, many mathematical researchers pay attention to how to find more accurate range and numerical methods of eigenvalues and eigenvectors of higher-order tensors. For example, there is a lot of literature on bounds and calculation

(c) The Author(s) 2019. This article is distributed under the terms of the Creative Commons Attribution 4.0 International License (http://creativecommons.org/licenses/by/4.0/), which permits unrestricted use, distribution, and reproduction in any medium, provided you give appropriate credit to the original author(s) and the source, provide a link to the Creative Commons license, and indicate if changes were made. 
methods of the spectral radius ( $H$-eigenvalue) of nonnegative tensors $[3,5,8,10,19,21$, 31, 34-36, 39, 43-46].

Equally important, the $Z$-eigenpair for nonnegative tensors plays a fundamental role in many applications such as high order Markov chains [13, 22], geometric measure of quantum entanglement [14], best rank-one approximation [6, 30, 47], and so on. Recently, due to the joint efforts of mathematicians, there are a series of theoretical conclusions and numerical methods to bound the $Z$-spectral radius for nonnegative tensors, these results are beneficial to further research and applications of the field.

In this paper, we mainly consider the bounds of $Z$-eigenpair of an irreducible nonnegative tensor. By estimating the ratio of the smallest and largest components of a Perron vector, we present some bounds for the eigenvector and $Z$-spectral radius of an irreducible and weakly symmetric nonnegative tensor. These proposed bounds extend and complement some existing ones. Furthermore, two examples are given to illustrate the proposed bounds.

This paper is organized as follows. In Sect. 2, we will give some basic facts and symbols. The concept of $Z$-eigenvalue and a Peron-Frobenius-type theorem is given [4]. In Sect. 3, we calculate the ratio of the smallest and largest components of a Perron vector. Moreover, a sharper bound of $Z$-spectral radius is shown for an irreducible and weakly symmetric nonnegative tensor. Two examples are given and the corresponding comparison is made intuitively and in detail. Some concluding remarks are presented in the final section.

\section{Preliminaries and basic facts}

For a positive integer $n, I_{n}$ denotes the set $I_{n}=\{1,2, \ldots, n\}$. Let $\mathbb{R}$ and $\mathbb{C}$ be the real and complex field, respectively. We call $\mathcal{A}=\left(a_{i_{1} i_{2} \cdots i_{m}}\right)$ a real (complex) tensor of $m$ th order and dimension $n$ if $a_{i_{1} i_{2} \cdots i_{m}} \in \mathbb{R}(\mathbb{C}), i_{1}, i_{2}, \ldots, i_{m} \in I_{n}$. Clearly, an $m$ th order $n$-dimensional tensor consists of $n^{m}$ entries from the real field $\mathbb{R}$. The set of all $m$ th order $n$-dimensional real tensors is denoted by $T_{m, n}$. For any tensor $\mathcal{A}=\left(a_{i_{1} \cdots i_{m}}\right) \in T_{m, n}$, if their entries $a_{i_{1} \cdots i_{m}}$ are invariant under any permutation of their indices, then $\mathcal{A}$ is called a symmetric tensor. We denote the set of all $m$ th order $n$-dimensional real symmetric tensors as $S_{m, n}$. Let $\pi(1,2, \ldots, n)$ be set of all permutations of $\{1,2, \ldots, n\}$. Let $\mathcal{A}=\left(a_{i_{1}} \ldots i_{m}\right) \in T_{m, n}$ and consider a vector $x=\left(x_{1}, x_{2}, \ldots, x_{n}\right)^{\top} \in \mathbb{R}^{n}$ or $\mathbb{C}^{n}$. Then $\mathcal{A} x^{m-1}$ is a vector with its $i$ th component defined by

$$
\left(\mathcal{A} x^{m-1}\right)_{i}:=\sum_{i_{2}, \ldots, i_{m}=1}^{n} a_{i i_{2} \cdots i_{m}} x_{i_{2}} \cdots x_{i_{m}}, \quad \forall i \in I_{n}
$$

and $\mathcal{A} x^{m}$ is a homogeneous polynomial of degree $m$,

$$
\mathcal{A} x^{m}:=x^{\top}\left(\mathcal{A} x^{m-1}\right)=\sum_{i_{1}, i_{2}, \ldots, i_{m}=1}^{n} a_{i_{1} i_{2} \cdots i_{m}} x_{i_{1}} x_{i_{2}} \cdots x_{i_{m}},
$$

where $x^{\top}$ is the transposition of $x$.

Definition 2.1 Let $\mathcal{A}=\left(a_{i_{1} i_{2} \cdots i_{m}}\right) \in T_{m, n}$. We call a number $\lambda \in \mathbb{C}$ an $E$-eigenvalue of $\mathcal{A}$ if there is a nonzero vector $x \in \mathbb{C}^{n}$ which solves the following system of polynomial equa- 
tions:

$$
\begin{aligned}
& \mathcal{A} x^{m-1}=\lambda x, \\
& x^{T} x=1,
\end{aligned}
$$

and call the solution $x$ an $E$-eigenvector of $\mathcal{A}$ associated with the eigenvalue $\lambda$. Any such pair $(\lambda, x)$ is called an $E$-eigenpair of $\mathcal{A}$. We call $(\lambda, x)$ a $Z$-eigenpair if they are both real.

Definition 2.2 The set of all $Z$-eigenvalues of $\mathcal{A}$ is called the $Z$-spectrum of $\mathcal{A}$, denoted as $\sigma_{z}(\mathcal{A})$. The largest modulus of the elements in the $Z$-spectrum of $\mathcal{A}$ is called the $Z$-spectral radius of $\mathcal{A}$, denoted as $\rho_{z}(\mathcal{A})$.

Definition 2.3 For any given tensor $\mathcal{A}=\left(a_{i_{1} i_{2} \cdots i_{m}}\right) \in T_{m, n}$, we say that $\mathcal{A}$ is reducible if there exists a nonempty proper index subset $J \subset I_{n}$ such that

$$
a_{i_{1} \cdots i_{m}}=0, \quad \forall i_{1} \in J, \forall i_{2}, \ldots, i_{m} \notin J
$$

$\mathcal{A}$ is called irreducible if it is not reducible.

Definition 2.4 A real tensor $\mathcal{A}$ is called weakly symmetric if the associated homogeneous polynomial

$$
f_{\mathcal{A}}(x):=\mathcal{A} x^{m}=\sum_{i_{2}, \ldots, i_{m}=1}^{n} a_{i_{1} i_{2} \cdots i_{m}} x_{i_{1}} x_{i_{2}} \cdots x_{i_{m}}
$$

satisfies $\nabla f_{\mathcal{A}}(x)=m \mathcal{A} x^{m-1}$.

An $m$ th order $n$-dimensional tensor $\mathcal{A}$ is called nonnegative (or, respectively, positive) if $a_{i_{1} \cdots i_{m}} \geq 0$ (or, respectively, $a_{i_{1} \cdots i_{m}}>0$ ) for all $i_{1}, \ldots, i_{m} \in I_{n}$. We denote the set of all nonnegative (or, respectively, positive) tensors of $m$ th order and dimension $n$ by $\mathbb{R}_{+}^{[m, n]}$ (or, respectively, $\mathbb{R}_{++}^{[m, n]}$ ).

Theorem 2.1 ([4]) Let $\mathcal{A}$ be an mth order $n$-dimensional nonnegative tensor. Then

(i) There exists a $Z$-eigenvalue $\lambda_{0} \geq 0$ of $\mathcal{A}$ with a nonnegative $Z$-eigenvector $x_{0} \neq 0$, i.e.,

$$
\mathcal{A} x_{0}^{m-1}=\lambda_{0} x_{0}, \quad x_{0}^{\top} x_{0}=1
$$

(ii) The above $Z$-eigenvalue $\lambda_{0}$ and its $Z$-eigenvector $x_{0}$ are positive if $\mathcal{A}$ is irreducible;

(iii) The $Z$-spectral radius $\rho_{z}(A)$ is a positive $Z$-eigenvalue with a positive $Z$-eigenvector if $\mathcal{A}$ is weakly symmetric and irreducible.

Recently, there appeared a series of theoretical conclusions and numerical methods to bound the $Z$-spectral radius for nonnegative tensors. For instance, Chang, Pearson and Zhang [4] studied some variation principles of $Z$-eigenvalues of nonnegative tensors. As a corollary of the main results, they presented the lower bound of $Z$-spectral radius for irreducible weakly symmetric nonnegative tensors (see Corollary 4.10 of [4]) as follows:

$$
\max \left\{c_{1}, c_{2}\right\} \leq \rho_{z}(\mathcal{A}),
$$


where $c_{1}=\max _{i} a_{i \ldots i}$ and $c_{2}=\left(\frac{1}{\sqrt{n}}\right)^{m-2} \min _{i} \sum_{i_{2}, \ldots, i_{m}=1}^{n} a_{i i_{2} \ldots i_{m}}$. For a nonnegative tensor, they also gave an upper bound for the $Z$-spectral radius (see Proposition 3.3 of [4]):

$$
\rho_{z}(\mathcal{A}) \leq \sqrt{n} \max _{i} \sum_{i_{2}, \ldots, i_{m}=1}^{n} a_{i i_{2} \ldots i_{m}}
$$

Song and Qi [34] proved a sharper upper bound for the $Z$-spectral radius of any $m$ th order $n$-dimensional tensor (see Corollary 4.5 of [34]):

$$
\rho_{z}(\mathcal{A}) \leq \max _{i} \sum_{i_{2}, \ldots, i_{m}=1}^{n}\left|a_{i i_{2} \ldots i_{m}}\right|
$$

He and Huang [11] obtained an upper bound of the $Z$-spectral radius for a weakly symmetric positive tensor (see Theorem 2.7 of [11]):

$$
\rho_{z}(\mathcal{A}) \leq R-l(1-\theta)
$$

where $r_{i}=\sum_{i_{2}, \ldots, i_{m}=1}^{n} a_{i i_{2} \ldots i_{m}}, R=\max _{i} r_{i}, r=\min _{i} r_{i}, l=\min _{i_{1}, \ldots, i_{m}} a_{i_{1} \ldots i_{m}}$, and $\theta=\left(\frac{r}{R}\right)^{\frac{1}{m}}$.

$\mathrm{Li}$, Liu and Vong [20] gave an upper bound of the $Z$-spectral radius for any tensor:

$$
\rho_{z}(\mathcal{A}) \leq \min _{k \in[m]} \max _{i_{k}} \sum_{i_{t}=1, t \in[m] \backslash\{k\}}^{n}\left|a_{i_{1} \cdots i_{k} \cdots i_{m}}\right|
$$

Moreover, they also presented two-sided bounds of the $Z$-spectral radius for an irreducible weakly symmetric nonnegative tensor:

$$
d_{m, n} \leq \rho_{z}(\mathcal{A}) \leq \max _{i, j}\left\{r_{i}+a_{i j \cdots j}\left(\delta^{-\frac{m-1}{m}}-1\right)\right\}
$$

where $\delta=\frac{\min _{i, j} a_{i j \cdots j}}{r-\min _{i j} a_{i j \cdots j}}\left(\gamma^{\frac{m-1}{m}}-\gamma^{\frac{1}{m}}\right)+\gamma, \gamma=\frac{R-\min _{i, j} a_{i j \cdots j}}{r-\min _{i, j} a_{i j \cdots j}}, r_{i}=\sum_{i_{2}, \ldots, i_{m}=1}^{n} a_{i i_{2} \ldots i_{m}}, R=\max _{i} r_{i}$, $r=\min _{i} r_{i}$, and

$$
d_{m, n}=\max _{k \in[m] \backslash\{1\}} \min _{i_{1}}\left[\left(\delta^{\frac{1}{m}}-1\right) \min _{i_{t}, t \in[m] \backslash\{1\}} a_{i_{1} \cdots i_{k} \cdots i_{m}}+\min _{i_{t}, t \in[m] \backslash\{1, k\}} \sum_{i_{k}=1}^{n} a_{i_{1} \cdots i_{k} \cdots i_{m}}\right] .
$$

Recently, Li, Liu and Vong [21] obtained an upper bound of the $Z$-spectral radius for an irreducible weakly symmetric nonnegative tensor by the following equation: for a Perron vector $x=\left(x_{1}, \ldots, x_{n}\right)^{\top}$,

$$
\frac{x_{\max }}{x_{\min }} \geq \eta(\mathcal{A})^{\frac{1}{m}}
$$

and

$$
\rho_{z}(\mathcal{A}) \leq \max _{i, j \in I_{n}}\left(\sum_{k=0}^{m-1} \mathcal{A}_{i, \alpha(k, j)} \eta^{-\frac{k}{m}}\right)
$$


where $x_{\min }=\min _{1 \leq i \leq n} x_{i}, x_{\max }=\max _{1 \leq i \leq n} x_{i}$,

$$
\begin{aligned}
\eta(\mathcal{A}) & =\frac{\sum_{k=t}^{m-1} \min _{i, j \in I_{n}} \mathcal{A}_{i, \alpha(k, j)}\left[\gamma^{\frac{k}{m}}-\gamma^{\frac{m-k}{m}}\right]+\max _{i \in I_{n}} r_{i}-\sum_{k=t}^{m-1} \min _{i, j \in I_{n}} \mathcal{A}_{i, \alpha(k, j)}}{\min _{i \in I_{n}} r_{i}-\sum_{k=1}^{t-1} \min _{i, j \in I_{n}} \mathcal{A}_{i, \alpha(k, j)}\left(1-\gamma^{-\frac{k}{m}}\right)-\sum_{k=t}^{m-1} \min _{i, j \in I_{n}} \mathcal{A}_{i, \alpha(k, j)}}, \\
\mathcal{A}_{i, \alpha(k, j)} & \sum_{\substack{\left.s_{1}<\cdots<s_{k} \\
s_{k+1}<\cdots<s_{m-1} \\
s_{1}, \ldots, s_{k}, \ldots, s_{m-1}\right\} \in \pi(2, \ldots, m)}} \sum_{\begin{array}{c}
i_{s_{1}}=\cdots=i_{s_{k}}=j \\
i_{k+1}=\cdots=i_{s_{m-1}} \neq j
\end{array}} a_{i_{1} i_{2} \cdots i_{m}}, \quad 0 \leq k \leq m-1,
\end{aligned}
$$

$\gamma=\frac{\max _{i \in I_{n}} r_{i}-\min _{i, j} a_{i j \cdots j}}{\min _{i \in I_{n}} r_{i}-\min _{i, j} a_{i j \ldots j}}, r_{i}=\sum_{i_{2}, \ldots, i_{m}=1}^{n} a_{i i_{2} \ldots i_{m}}$, and $t=\left[\frac{m}{2}\right]$. From (2.8), they have the following conclusion:

$$
\rho_{z}(\mathcal{A}) \leq \max _{i, j \in I_{n}}\left(\sum_{k=0}^{m-1} \mathcal{S}_{i, \alpha(k, j)}^{\prime} \eta^{-\frac{k}{m}}\right),
$$

where $\mathcal{S}^{\prime}=\frac{1}{m !} \mathcal{S}, \eta \equiv \eta\left(\mathcal{S}^{\prime}\right), \mathcal{S}=\left(s_{i_{1} \cdots i_{m}}\right) \in R^{[m, n]}$, and $s_{i_{1} \cdots i_{m}}=\sum_{\left(j_{1}, \ldots, j_{m}\right) \in \pi\left(i_{1}, \ldots, i_{m}\right)} a_{j_{1} \cdots j_{m}}$. However, there is a small negligence here since they use $t \geq m-t$ in their proof, but the fact that $t=\left[\frac{m}{2}\right]$ may not imply $t \geq m-t$ (for example, for $m=3, t=\left[\frac{m}{2}\right]=1$ and $m-t=2$ ). In this paper, we will modify this negligence by taking $t=m-\left[\frac{m}{2}\right]$.

Obviously, the bound (2.5) is sharper than those in (2.2) and (2.3) for any tensor. Since $\delta \geq 1$, it's easy to see that the upper bound in (2.6) is sharper than that in (2.4) when the tensor is assumed to be weakly symmetric positive. Since $\eta(\mathcal{A}) \geq \delta \geq \gamma \geq 1$, hence the upper bound in (2.8) is always better than that in (2.6). When the tensor is irreducible symmetric nonnegative, the bound in (2.9) becomes that in (2.8).

\section{Bounds for the Z-spectral radius of nonnegative tensors}

Theorem 3.1 Let $\mathcal{A}=\left(a_{i_{1} i_{2} \cdots i_{m}}\right) \in \mathbb{R}^{[m, n]}$ be a nonnegative tensor having a positive $Z$ eigenpair. Then for any $Z$-eigenpair $(\lambda, x)$ of $\mathcal{A}$ with a positive $Z$-eigenvector $x$, we have

$$
\frac{x_{\max }}{x_{\min }} \geq \varphi(\mathcal{A})^{\frac{1}{m}}
$$

where $x_{s}=x_{\min }=\min _{i \in I_{n}} x_{i}, x_{l}=x_{\max }=\max _{i \in I_{n}} x_{i}$,

$$
\begin{aligned}
& \varphi(\mathcal{A})=\frac{\sum_{k=t}^{m-1}\left(\begin{array}{c}
m-1 \\
m-k-1
\end{array}\right)(n-1)^{m-k-1} \beta_{m-k-1}(\mathcal{A})\left[\gamma^{\frac{k}{m}}-\gamma^{\frac{m-k}{m}}\right]+R-\sum_{k=t}^{m-1}\left(\begin{array}{c}
m-1 \\
m-k-1
\end{array}\right)(n-1)^{m-k-1} \beta_{m-k-1}(\mathcal{A})}{r-\sum_{k=1}^{t-1}\left(\begin{array}{c}
m-1 \\
m-k-1
\end{array}\right)(n-1)^{m-k-1} \beta_{m-k-1}(\mathcal{A})\left(1-\gamma^{-\frac{k}{m}}\right)-\sum_{k=t}^{m-1}\left(\begin{array}{c}
m-1 \\
m-k-1
\end{array}\right)(n-1)^{m-k-1} \beta_{m-k-1}(\mathcal{A})}, \\
& \beta_{t}(\mathcal{A})=\min _{i, j \in I_{n}}\left\{a_{i i_{2} \cdots i_{m}}:\left(i_{2}, \ldots, i_{m}\right) \in \Delta(j, m-t-1)\right\}, \quad t=0,1, \ldots, m-2, \\
& \Delta(j, u)=\bigcup_{S \subseteq\{2, \ldots, m\}}\left\{\left(i_{2} \ldots, i_{m}\right): i_{v}=j, \forall v \in S, \text { and } i_{v} \neq j, \forall v \notin S\right\}, \quad u=0,1, \ldots, m-1, \\
& \gamma=\frac{R-\min _{i, j \in I_{n}} a_{i j \cdots j}}{r-\min _{i, j \in I_{n}} a_{i j \cdots j}}, \quad R=r_{p}=\max _{i, j \in I_{n}} r_{i}, r=r_{q}=\min _{i, j \in I_{n}} r_{i}, r_{i}=\sum_{i_{2} \cdots i_{m}=1}^{n} a_{i i_{2} \cdots i_{m}} .
\end{aligned}
$$

Proof According to Theorem 2.1, there exists an $x=\left(x_{1}, x_{2}, \ldots, x_{n}\right)^{T}>0$ such that $\mathcal{A} x^{m-1}=$ $\lambda x$. For $x_{s}=x_{\min }=\min _{i \in I_{n}} x_{i}$, it follows that

$$
\lambda x_{s} \leq \lambda x_{i}=\sum_{i_{2}, \ldots, i_{m} \in I_{n}} a_{i i_{2} \cdots i_{m}} x_{i_{2}} \cdots x_{i_{m}}
$$




$$
\begin{aligned}
& \leq a_{i s \cdots s} x_{s}^{m-1}+\sum_{\left(i_{2}, \ldots, i_{m}\right) \in \Delta(s, m-2)} a_{i i_{2} \cdots i_{m}} x_{s}^{m-2} x_{l}+\cdots \\
& +\sum_{\left(i_{2}, \ldots, i_{m}\right) \in \Delta(s, k)} a_{i i_{2} \cdots i_{m}} x_{s}^{k} x_{l}^{m-k-1}+\cdots \\
& +\sum_{\left(i_{2}, \ldots, i_{m}\right) \in \Delta(s, 1)} a_{i i_{2} \cdots i_{m}} x_{s} x_{l}^{m-2}+\sum_{\left(i_{2}, \ldots, i_{m}\right) \in \Delta(s, 0)} a_{i i_{2} \cdots i_{m}} x_{l}^{m-1} \\
& =a_{i s \cdots s}\left(x_{s}^{m-1}-x_{l}^{m-1}\right)+\sum_{\left(i_{2}, \ldots, i_{m}\right) \in \Delta(s, m-2)} a_{i i_{2} \cdots i_{m}}\left(x_{s}^{m-2} x_{l}-x_{l}^{m-1}\right)+\cdots \\
& +\sum_{\left(i_{2}, \ldots, i_{m}\right) \in \Delta(s, k)} a_{i i_{2} \cdots i_{m}}\left(x_{s}^{k} x_{l}^{m-k-1}-x_{l}^{m-1}\right)+\cdots \\
& +\sum_{\left(i_{2}, \ldots, i_{m}\right) \in \Delta(s, 1)} a_{i i_{2} \cdots i_{m}}\left(x_{s} x_{l}^{m-2}-x_{l}^{m-1}\right)+r_{i}(A) x_{l}^{m-1} \\
& \leq \min _{i, j \in I_{n}} a_{i j \cdots j}\left(x_{s}^{m-1}-x_{l}^{m-1}\right)+\left(\begin{array}{c}
m-1 \\
1
\end{array}\right)(n-1) \beta_{1}(\mathcal{A})\left(x_{s}^{m-2} x_{l}-x_{l}^{m-1}\right)+\cdots \\
& +\left(\begin{array}{l}
m-1 \\
m-2
\end{array}\right)(n-1)^{m-2} \beta_{m-2}(\mathcal{A})\left(x_{s} x_{l}^{m-2}-x_{l}^{m-1}\right)+r_{i}(\mathcal{A}) x_{l}^{m-1} \\
& =\sum_{k=0}^{m-2}\left(\begin{array}{c}
m-1 \\
k
\end{array}\right)(n-1)^{k} \beta_{k}(\mathcal{A})\left(x_{s}^{m-k-1} x_{l}^{k}-x_{l}^{m-1}\right)+r_{i}(\mathcal{A}) x_{l}^{m-1} .
\end{aligned}
$$

Taking $r_{i}=r_{q}=r$, since $x_{s}>0$, we have

$$
\begin{aligned}
\lambda \leq & \sum_{k=0}^{m-2}\left(\begin{array}{c}
m-1 \\
k
\end{array}\right)(n-1)^{k} \beta_{k}(\mathcal{A}) x_{s}^{m-k-2} x_{l}^{k} \\
& +\left(r-\sum_{k=0}^{m-2}\left(\begin{array}{c}
m-1 \\
k
\end{array}\right)(n-1)^{k} \beta_{k}(\mathcal{A})\right) \frac{x_{l}^{m-1}}{x_{s}} .
\end{aligned}
$$

For $x_{l}=x_{\max }=\max _{i \in I_{n}} x_{i}$, we similarly have

$$
\begin{aligned}
\lambda x_{l} \geq \lambda x_{i}= & \sum_{i_{2}, \ldots, i_{m} \in I_{n}} a_{i i_{2} \cdots i_{m}} x_{i_{2}} \cdots x_{i_{m}} \\
\geq & a_{i l \cdots l} x_{l}^{m-1}+\sum_{\left(i_{2}, \ldots, i_{m}\right) \in \Delta(l, m-2)} a_{i i_{2} \cdots i_{m}} x_{l}^{m-2} x_{s}+\cdots \\
& +\sum_{\left(i_{2}, \ldots, i_{m}\right) \in \Delta(l, k)} a_{i i_{2} \cdots i_{m}} x_{l}^{k} x_{s}^{m-k-1}+\cdots \\
& +\sum_{\left(i_{2}, \ldots, i_{m}\right) \in \Delta(l, 1)} a_{i i_{2} \cdots i_{m}} x_{l} x_{s}^{m-2}+\sum_{\left(i_{2}, \ldots, i_{m}\right) \in \Delta(l, 0)} a_{i i_{2} \cdots i_{m}} x_{s}^{m-1} \\
= & a_{i l \cdots l}\left(x_{l}^{m-1}-x_{s}^{m-1}\right)+\sum_{\left(i_{2}, \ldots, i_{m}\right) \in \Delta(l, m-2)} a_{i i_{2} \cdots i_{m}}\left(x_{l}^{m-2} x_{s}-x_{s}^{m-1}\right)+\cdots \\
& +\sum_{\left(i_{2}, \ldots, i_{m}\right) \in \Delta(l, k)} a_{i i_{2} \cdots i_{m}}\left(x_{l}^{k} x_{s}^{m-k-1}-x_{s}^{m-1}\right)+\cdots \\
& +\sum_{\left(i_{2}, \ldots, i_{m}\right) \in \Delta(l, 1)} a_{i i_{2} \cdots i_{m}}\left(x_{l} x_{s}^{m-2}-x_{s}^{m-1}\right)+r_{i}(\mathcal{A}) x_{s}^{m-1}
\end{aligned}
$$


Ma and Song Journal of Inequalities and Applications

(2019) 2019:271

Page 7 of 14

$$
\begin{aligned}
\geq & \min _{i, j \in I_{n}} a_{i j \ldots j}\left(x_{l}^{m-1}-x_{s}^{m-1}\right)+\left(\begin{array}{c}
m-1 \\
1
\end{array}\right)(n-1) \beta_{1}(\mathcal{A})\left(x_{l}^{m-2} x_{s}-x_{s}^{m-1}\right)+\cdots \\
& +\left(\begin{array}{c}
m-1 \\
m-2
\end{array}\right)(n-1)^{m-2} \beta_{m-2}(A)\left(x_{l} x_{s}^{m-2}-x_{s}^{m-1}\right)+r_{i}(\mathcal{A}) x_{s}^{m-1} \\
= & \sum_{k=0}^{m-2}\left(\begin{array}{c}
m-1 \\
k
\end{array}\right)(n-1)^{k} \beta_{k}(\mathcal{A})\left(x_{l}^{m-k-1} x_{s}^{k}-x_{s}^{m-1}\right)+r_{i}(\mathcal{A}) x_{s}^{m-1} .
\end{aligned}
$$

Taking $r_{i}=r_{p}$, since $x_{l}>0$, we have that

$$
\begin{aligned}
\lambda \geq & \sum_{k=0}^{m-2}\left(\begin{array}{c}
m-1 \\
k
\end{array}\right)(n-1)^{k} \beta_{k}(\mathcal{A}) x_{l}^{m-k-2} x_{s}^{k} \\
& +\left(R-\sum_{k=0}^{m-2}\left(\begin{array}{c}
m-1 \\
k
\end{array}\right)(n-1)^{k} \beta_{k}(\mathcal{A})\right) \frac{x_{s}^{m-1}}{x_{l}} .
\end{aligned}
$$

Combining (3.1) and (3.2) together gives

$$
\begin{aligned}
& \sum_{k=0}^{m-2}\left(\begin{array}{c}
m-1 \\
k
\end{array}\right)(n-1)^{k} \beta_{k}(\mathcal{A}) x_{l}^{m-k-2} x_{s}^{k}+\left(R-\sum_{k=0}^{m-2}\left(\begin{array}{c}
m-1 \\
k
\end{array}\right)(n-1)^{k} \beta_{k}(\mathcal{A})\right) \frac{x_{s}^{m-1}}{x_{l}} \\
& \leq \sum_{k=0}^{m-2}\left(\begin{array}{c}
m-1 \\
k
\end{array}\right)(n-1)^{k} \beta_{k}(\mathcal{A}) x_{s}^{m-k-2} x_{l}^{k} \\
& \quad+\left(r-\sum_{k=0}^{m-2}\left(\begin{array}{c}
m-1 \\
k
\end{array}\right)(n-1)^{k} \beta_{k}(\mathcal{A})\right) \frac{x_{l}^{m-1}}{x_{s}} .
\end{aligned}
$$

Multiplying by $\frac{x_{l}}{x_{s}^{m-1}}$ on both sides gives

$$
\begin{aligned}
& \sum_{k=0}^{m-2}\left(\begin{array}{c}
m-1 \\
k
\end{array}\right)(n-1)^{k} \beta_{k}(\mathcal{A}) \frac{x_{l}^{m-k-2} x_{s}^{k} x_{l}}{x_{s}^{m-1}}+\left(R-\sum_{k=0}^{m-2}\left(\begin{array}{c}
m-1 \\
k
\end{array}\right)(n-1)^{k} \beta_{k}(\mathcal{A})\right) \\
& \leq \sum_{k=0}^{m-2}\left(\begin{array}{c}
m-1 \\
k
\end{array}\right)(n-1)^{k} \beta_{k}(\mathcal{A}) \frac{x_{s}^{m-k-2} x_{l}^{k} x_{l}}{x_{s}^{m-1}} \\
& \quad+\left(r-\sum_{k=0}^{m-2}\left(\begin{array}{c}
m-1 \\
k
\end{array}\right)(n-1)^{k} \beta_{k}(\mathcal{A})\right)\left(\frac{x_{l}}{x_{s}}\right)^{m},
\end{aligned}
$$

and so we have

$$
\begin{aligned}
& \sum_{k=0}^{m-2}\left(\begin{array}{c}
m-1 \\
k
\end{array}\right)(n-1)^{k} \beta_{k}(\mathcal{A})\left(\frac{x_{l}}{x_{s}}\right)^{m-k-1}+\left(R-\sum_{k=0}^{m-2}\left(\begin{array}{c}
m-1 \\
k
\end{array}\right)(n-1)^{k} \beta_{k}(\mathcal{A})\right) \\
& \leq \sum_{k=0}^{m-2}\left(\begin{array}{c}
m-1 \\
k
\end{array}\right)(n-1)^{k} \beta_{k}(\mathcal{A})\left(\frac{x_{l}}{x_{s}}\right)^{k+1} \\
& \quad+\left(r-\sum_{k=0}^{m-2}\left(\begin{array}{c}
m-1 \\
k
\end{array}\right)(n-1)^{k} \beta_{k}(\mathcal{A})\right)\left(\frac{x_{l}}{x_{s}}\right)^{m} .
\end{aligned}
$$


Since $\left(\frac{x_{l}}{x_{s}}\right)^{m} \geq\left(\frac{x_{l}}{x_{s}}\right)^{m-1} \geq \cdots \geq \frac{x_{l}}{x_{s}} \geq 1$, by (3.3), we get

$$
\begin{aligned}
& \min _{i, j \in I_{n}} a_{i j \ldots j}\left(\frac{x_{l}}{x_{s}}\right)^{m-1}+\sum_{k=1}^{m-2}\left(\begin{array}{c}
m-1 \\
k
\end{array}\right)(n-1)^{k} \beta_{k}(A)+\left(R-\sum_{k=0}^{m-2}\left(\begin{array}{c}
m-1 \\
k
\end{array}\right)(n-1)^{k} \beta_{k}(\mathcal{A})\right) \\
& \leq \min _{i, j \in I_{n}} a_{i j \cdots j} \frac{x_{l}}{x_{s}}+\sum_{k=1}^{m-2}\left(\begin{array}{c}
m-1 \\
k
\end{array}\right)(n-1)^{k} \beta_{k}(\mathcal{A})\left(\frac{x_{l}}{x_{s}}\right)^{m} \\
& +\left(r-\sum_{k=0}^{m-2}\left(\begin{array}{c}
m-1 \\
k
\end{array}\right)(n-1)^{k} \beta_{k}(A)\right)\left(\frac{x_{l}}{x_{s}}\right)^{m},
\end{aligned}
$$

i.e.,

$$
\min _{i, j \in I_{n}} a_{i j \cdots j}\left(\frac{x_{l}}{x_{s}}\right)^{m-1}+\left(R-\min _{i, j \in I_{n}} a_{i j \cdots j}\right) \leq \min _{i, j \in I_{n}} a_{i j \cdots j} \frac{x_{l}}{x_{s}}+\left(r-\min _{i, j \in I_{n}} a_{i j \cdots j}\right)\left(\frac{x_{l}}{x_{s}}\right)^{m} .
$$

Hence

$$
\left(\frac{x_{l}}{x_{s}}\right)^{m} \geq \frac{R-\min _{i, j \in I_{n}} a_{i j \cdots j}}{r-\min _{i, j \in I_{n}} a_{i j \cdots j}}
$$

Let $\gamma=\frac{R-\min _{i, j \in I_{n}} a_{i j \cdots j}}{r-\min _{i, j \in I_{n}} a_{i j \cdots j}}$. Then $\frac{x_{l}}{x_{s}} \geq \gamma^{\frac{1}{m}} \geq 1$. Let $t=m-\left[\frac{m}{2}\right]$. Then $t \geq m-t$, so by (3.3) again, we have

$$
\begin{aligned}
\min _{i, j \in I_{n}} & a_{i j \ldots j}\left(\frac{x_{l}}{x_{s}}\right)^{m-1}+\left(\begin{array}{c}
m-1 \\
1
\end{array}\right)(n-1) \beta_{1}(\mathcal{A})\left(\frac{x_{l}}{x_{s}}\right)^{m-2}+\cdots \\
& +\left(\begin{array}{c}
m-1 \\
m-t-1
\end{array}\right)(n-1)^{m-t-1} \beta_{m-t-1}(\mathcal{A})\left(\frac{x_{l}}{x_{s}}\right)^{t} \\
& +\left(R-\sum_{k=t}^{m-1}\left(\begin{array}{c}
m-1 \\
m-k-1
\end{array}\right)(n-1)^{m-k-1} \beta_{m-k-1}(\mathcal{A})\right) \\
\leq & \min _{i, j \in I_{n}} a_{i j \cdots j} \frac{x_{l}}{x_{s}}+\left(\begin{array}{c}
m-1 \\
1
\end{array}\right)(n-1) \beta_{1}(A)\left(\frac{x_{l}}{x_{s}}\right)^{2}+\cdots \\
& +\left(\begin{array}{c}
m-1 \\
m-t-1
\end{array}\right)(n-1)^{m-t-1} \beta_{m-t-1}(\mathcal{A})\left(\frac{x_{l}}{x_{s}}\right)^{m-t} \\
& +\left(\begin{array}{l}
m-1 \\
m-t
\end{array}\right)(n-1)^{m-t} \beta_{m-t}(\mathcal{A})\left(\frac{x_{l}}{x_{s}}\right)^{m} \gamma^{-\frac{t-1}{m}}+\cdots \\
+ & \left(\begin{array}{l}
m-1 \\
m-2
\end{array}\right)(n-1)^{m-2} \beta_{m-2}(\mathcal{A})\left(\frac{x_{l}}{x_{s}}\right)^{m} \gamma^{-\frac{1}{m}} \\
+ & \left(\begin{array}{l}
m-2 \\
r-\sum_{k=0}^{m}(m-1 \\
m-1
\end{array}\right)\left(\begin{array}{l}
x_{l} \\
x_{s}
\end{array}\right)^{m}(\mathcal{A})
\end{aligned}
$$




$$
\begin{aligned}
& =\min _{i, j \in I_{n}} a_{i j \ldots j}\left(\frac{x_{l}}{x_{s}}\right)+\left(\begin{array}{c}
m-1 \\
1
\end{array}\right)(n-1) \beta_{1}(\mathcal{A})\left(\frac{x_{l}}{x_{s}}\right)^{2}+\cdots \\
& +\left(\begin{array}{c}
m-1 \\
m-t-1
\end{array}\right)(n-1)^{m-t-1} \beta_{m-t-1}(\mathcal{A})\left(\frac{x_{l}}{x_{s}}\right)^{m-t} \\
& +\left[r-\sum_{k=1}^{t-1}\left(\begin{array}{c}
m-1 \\
m-k-1
\end{array}\right)(n-1)^{m-k-1} \beta_{m-k-1}(\mathcal{A})\left(1-\gamma^{-\frac{k}{m}}\right)\right. \\
& \left.-\sum_{k=t}^{m-1}\left(\begin{array}{c}
m-1 \\
m-k-1
\end{array}\right)(n-1)^{m-k-1} \beta_{m-k-1}(\mathcal{A})\right]\left(\frac{x_{l}}{x_{s}}\right)^{m} \text {. }
\end{aligned}
$$

Since $\frac{x_{l}}{x_{s}} \geq \gamma^{\frac{1}{m}} \geq 1$ and $t \geq m-t$, we have

$$
\begin{aligned}
& {\left[r-\sum_{k=1}^{t-1}\left(\begin{array}{c}
m-1 \\
m-k-1
\end{array}\right)(n-1)^{m-k-1} \beta_{m-k-1}(\mathcal{A})\left(1-\gamma^{-\frac{k}{m}}\right)\right.} \\
& \left.-\sum_{k=t}^{m-1}\left(\begin{array}{c}
m-1 \\
m-k-1
\end{array}\right)(n-1)^{m-k-1} \beta_{m-k-1}(\mathcal{A})\right]\left(\frac{x_{l}}{x_{s}}\right)^{m} \\
& \geq \min _{i, j \in I_{n}} a_{i j \ldots j}\left[\left(\frac{x_{l}}{x_{s}}\right)^{m-1}-\frac{x_{l}}{x_{s}}\right]+\left(\begin{array}{c}
m-1 \\
1
\end{array}\right)(n-1) \beta_{1}(\mathcal{A})\left[\left(\frac{x_{l}}{x_{s}}\right)^{m-2}-\left(\frac{x_{l}}{x_{s}}\right)^{2}\right]+\cdots \\
& +\left(\begin{array}{c}
m-1 \\
m-t-1
\end{array}\right)(n-1)^{m-t-1} \beta_{m-t-1}(\mathcal{A})\left[\left(\frac{x_{l}}{x_{s}}\right)^{t}-\left(\frac{x_{l}}{x_{s}}\right)^{m-t}\right] \\
& +\left(R-\sum_{k=t}^{m-1}\left(\begin{array}{c}
m-1 \\
m-k-1
\end{array}\right)(n-1)^{m-k-1} \beta_{m-k-1}(\mathcal{A})\right) \\
& \geq \min _{i, j \in I_{n}} a_{i j \ldots j}\left[\gamma^{\frac{m-1}{m}}-\gamma^{\frac{1}{m}}\right]+\left(\begin{array}{c}
m-1 \\
1
\end{array}\right)(n-1) \beta_{1}(A)\left[\gamma^{\frac{m-2}{m}}-\gamma^{\frac{2}{m}}\right]+\cdots \\
& +\left(\begin{array}{c}
m-1 \\
m-t-1
\end{array}\right)(n-1)^{m-t-1} \beta_{m-t-1}(\mathcal{A})\left[\gamma^{\frac{t}{m}}-\gamma^{\frac{m-t}{m}}\right] \\
& +\left(R-\sum_{k=t}^{m-1}\left(\begin{array}{c}
m-1 \\
m-k-1
\end{array}\right)(n-1)^{m-k-1} \beta_{m-k-1}(\mathcal{A})\right),
\end{aligned}
$$

and hence, $\frac{x_{l}}{x_{s}} \geq(\varphi(\mathcal{A}))^{\frac{1}{m}}$, where

$$
\varphi(\mathcal{A})=\frac{\sum_{k=t}^{m-1}\left(\begin{array}{c}
m-1 \\
m-k-1
\end{array}\right)(n-1)^{m-k-1} \beta_{m-k-1}(\mathcal{A})\left[\gamma^{\frac{k}{m}}-\gamma^{\frac{m-k}{m}}\right]+R-\sum_{k=t}^{m-1}\left(\begin{array}{c}
m-1 \\
m-k-1
\end{array}\right)(n-1)^{m-k-1} \beta_{m-k-1}(\mathcal{A})}{r-\sum_{k=1}^{t-1}\left(\begin{array}{c}
m-1 \\
m-k-1
\end{array}\right)(n-1)^{m-k-1} \beta_{m-k-1}(\mathcal{A})\left(1-\gamma^{-\frac{k}{m}}\right)-\sum_{k=t}^{m-1}\left(\begin{array}{c}
m-1 \\
m-k-1
\end{array}\right)(n-1)^{m-k-1} \beta_{m-k-1}(\mathcal{A})} .
$$

From Theorem 3.1, we have the following upper bound. 
Theorem 3.2 Let $A \in \mathbb{R}^{[m, n]}$ be an irreducible and weakly symmetric nonnegative tensor. Then we have

$$
\rho_{z}(A) \leq \max _{i, j \in I_{n}}\left(\sum_{k=0}^{m-1} \sum_{\left(i_{2}, \ldots, i_{m}\right) \in \Delta(j, k)} a_{i i_{2} \cdots i_{m}} \varphi^{-\frac{k}{m}}\right),
$$

where $\varphi \equiv \varphi(A)$ is given in Theorem 3.1.

Proof Since $\mathcal{A}$ is a weakly irreducible nonnegative symmetric tensor, we know that the $Z$ spectral radius $\rho \equiv \rho_{z}(A)$ is a positive $Z$-eigenvalue with a positive $Z$-eigenvector $x$. Since $x^{T} x=1$ and $x>0$, then $x_{i}^{m-1} \leq x_{i}$ for any $i$. Let $x_{s}=x_{\min }=\min _{i \in I_{n}} x_{i}, x_{l}=x_{\max }=\min _{i \in I_{n}} x_{i}$. Then we have

$$
\begin{aligned}
\rho(\mathcal{A}) x_{i}^{m-1} \leq & \rho(\mathcal{A}) x_{i} \\
\leq & a_{i s \cdots s} x_{s}^{m-1}+\sum_{\left(i_{2}, \ldots, i_{m}\right) \in \Delta(s, m-2)} a_{i i_{2} \cdots i_{m}} x_{s}^{m-2} x_{l}+\cdots \\
& +\sum_{\left(i_{2}, \ldots, i_{m}\right) \in \Delta(s, 1)} a_{i i_{2} \cdots i_{m}} x_{s} x_{l}^{m-2}+\sum_{\left(i_{2}, \ldots, i_{m}\right) \in \Delta(s, 0)} a_{i i_{2} \cdots i_{m}} x_{l}^{m-1} .
\end{aligned}
$$

Taking $i=l$ and multiplying by $x_{l}^{1-m}$ on both sides of the above inequality, from Theorem 3.1, we get

$$
\begin{aligned}
\rho(\mathcal{A}) \leq & a_{i s \cdots s}\left(\frac{x_{s}}{x_{l}}\right)^{m-1}+\sum_{\left(i_{2}, \ldots, i_{m}\right) \in \Delta(s, m-2)} a_{i i_{2} \cdots i_{m}}\left(\frac{x_{s}}{x_{l}}\right)^{m-2}+\cdots \\
& +\sum_{\left(i_{2}, \ldots, i_{m}\right) \in \Delta(s, 1)} a_{i i_{2} \cdots i_{m}} \frac{x_{s}}{x_{l}}+\sum_{\left(i_{2}, \ldots, i_{m}\right) \in \Delta(s, 0)} a_{i i_{2} \cdots i_{m}} \\
\leq & \max _{i, j \in I_{n}}\left\{a_{i j \cdots j} \varphi^{-\frac{m-1}{m}}+\sum_{\left(i_{2}, \ldots, i_{m}\right) \in \Delta(j, m-2)} a_{i i_{2} \cdots i_{m}} \varphi^{-\frac{m-2}{m}}+\cdots\right. \\
& \left.+\sum_{\left(i_{2}, \ldots, i_{m}\right) \in \Delta(j, 1)} a_{i i_{2} \cdots i_{m}} \varphi^{-\frac{1}{m}}+\sum_{\left(i_{2}, \ldots, i_{m}\right) \in \Delta(j, 0)} a_{i i_{2} \cdots i_{m}}\right\} \\
= & \max _{i, j \in I_{n}}\left(\sum_{k=0}^{m-1} \sum_{\left(i_{2}, \ldots, i_{m}\right) \in \Delta(j, k)} a_{i i_{2} \cdots i_{m}} \varphi^{-\frac{k}{m}}\right) .
\end{aligned}
$$

Remark 3.1 For the matrix case $A=\left(a_{i j}\right) \in \mathbb{R}^{[n \times n]}, i, j \in I_{n}$, i.e., when $m=2$, it is easy to see that the bound in Theorem 3.2 reduces to the following one:

$$
\rho_{z}(A) \leq \max _{i, j}\left\{r_{i}(A)+a_{i j}(\xi-1)\right\}
$$

where $\xi=\left(\frac{R-\min _{i j} a_{i j}}{r-\min _{i j} a_{i j}}\right)^{-\frac{1}{2}}, R=\max _{i} r_{i}, r=\min _{i} r_{i}$, and $r_{i}=\sum_{j=1}^{n} a_{i j}$, which is the same as the bound in (2.6) for the matrix case. 
Remark 3.2 Let $\delta=\frac{a}{c}$ and $\varphi=\frac{a+b}{c-d}$, where $\delta$ is given by (2.6), $\varphi$ is given by Theorem 3.1 and

$$
\left\{\begin{aligned}
a= & \min _{i, j \in I_{n}} a_{i j \ldots j}\left(\gamma^{\frac{m-1}{m}}-\gamma^{\frac{1}{m}}\right)+R-\min _{i, j \in I_{n}} a_{i j \cdots j} \\
b= & \sum_{k=t}^{m-2}\left(\begin{array}{c}
m-1 \\
m-k-1
\end{array}\right)(n-1)^{m-k-1} \beta_{m-k-1}(\mathcal{A})\left(\gamma^{\frac{k}{m}}-\gamma^{\frac{m-k}{m}}\right) \\
& -\sum_{k=t}^{m-2}\left(\begin{array}{c}
m-1 \\
m-k-1
\end{array}\right)(n-1)^{m-k-1} \beta_{m-k-1}(\mathcal{A}) \\
c= & r-\min _{i, j \in I_{n}} a_{i j \cdots j} \\
d= & \sum_{k=1}^{t-1}\left(\begin{array}{c}
m-1 \\
m-k-1
\end{array}\right)(n-1)^{m-k-1} \beta_{m-k-1}(\mathcal{A})\left(1-\gamma^{-\frac{k}{m}}\right) \\
& +\sum_{k=t}^{m-2}\left(\begin{array}{c}
m-1 \\
m-k-1
\end{array}\right)(n-1)^{m-k-1} \beta_{m-k-1}(\mathcal{A})
\end{aligned}\right.
$$

Since $\delta \geq \gamma \geq 1$ and $a, c, d, a+b, c-d \geq 0$, it's easy to conclude that

$$
\begin{aligned}
\frac{b}{d} & \geq-\frac{\sum_{k=t}^{m-2}\left(\begin{array}{c}
m-1 \\
m-k-1
\end{array}\right)(n-1)^{m-k-1} \beta_{m-k-1}(\mathcal{A})}{\sum_{k=1}^{t-1}\left(\begin{array}{c}
m-1 \\
m-k-1
\end{array}\right)(n-1)^{m-k-1} \beta_{m-k-1}(\mathcal{A})\left(1-\gamma^{-\frac{k}{m}}\right)+\sum_{k=t}^{m-2}\left(\begin{array}{c}
m-1 \\
m-k-1
\end{array}\right)(n-1)^{m-k-1} \beta_{m-k-1}(\mathcal{A})} \\
& \geq-1 \geq-\delta=-\frac{a}{c},
\end{aligned}
$$

when $d>0$, and $b=\sum_{k=t}^{m-2}\left(\begin{array}{c}m-1 \\ m-k-1\end{array}\right)(n-1)^{m-k-1} \beta_{m-k-1}(\mathcal{A})\left(\gamma^{\frac{k}{m}}-\gamma^{\frac{m-k}{m}}\right) \geq 0$, when $d=0$.

Then we have $\frac{a+b}{c-d} \geq \frac{a}{c}$, i.e., $\varphi \geq \delta \geq \gamma \geq 1$.

Thus the upper bound in Theorem 3.2 is better than that in (2.6).

The authors presented the following bound in [20]:

$$
\frac{x_{\max }}{x_{\min }} \geq \delta^{\frac{1}{m}}
$$

where

$$
\delta=\frac{\min _{i, j \in I_{n}} a_{i j \cdots j}}{r-\min _{i, j \in I_{n}} a_{i j \cdots j}}\left(\gamma^{\frac{m-1}{m}}-\gamma^{\frac{1}{m}}\right)+\gamma, \quad \gamma=\frac{R-\min _{i, j \in I_{n}} a_{i j \ldots j}}{r-\min _{i, j \in I_{n}} a_{i j \cdots j}} .
$$

Example 3.1 Let $\mathcal{A}=\left(a_{i_{1} i_{2} i_{3}}\right) \in \mathbb{R}_{+}^{[3,2]}$ with

$$
a_{111}=a_{121}=k, \quad a_{112}=a_{222}=1, \quad a_{211}=a_{212}=a_{221}=a_{122}=2, \quad k>4 .
$$

Then $\mathcal{A}$ is a positive tensor. A simple computation with (3.5) gives $R=2 k+3, r=7, \gamma=\frac{k+1}{3}$, and then $\delta=\frac{1}{6}\left[\left(\frac{k+1}{3}\right)^{\frac{2}{3}}-\left(\frac{k+1}{3}\right)^{\frac{1}{3}}\right]+\frac{k+1}{3}$. However, by Theorem 3.1, we have $t=2, \beta_{0}(\mathcal{A})=$ $\min _{i, j} a_{i j \ldots j}=1, \beta_{1}(\mathcal{A})=1$, thus

$$
\varphi=\frac{\left[\left(\frac{k+1}{3}\right)^{\frac{2}{3}}-\left(\frac{k+1}{3}\right)^{\frac{1}{3}}\right]+2 k+3-1}{7-2\left[1-\left(\frac{k+1}{3}\right)^{-\frac{1}{3}}\right]-1}=\frac{\left[\left(\frac{k+1}{3}\right)^{\frac{2}{3}}-\left(\frac{k+1}{3}\right)^{\frac{1}{3}}\right]+2 k+2}{4+2\left(\frac{k+1}{3}\right)^{-\frac{1}{3}}} .
$$

Now we take $k=10$, and then in (3.5) we have

$$
1.5613 \leq \frac{x_{\max }}{x_{\min }}
$$

while in Theorem 3.1,

$$
1.6275 \leq \frac{x_{\max }}{x_{\min }} .
$$


Remark 3.3 Let $\eta=\frac{e}{g}$ and $\varphi=\frac{f}{h}$, where $\eta$ is given by (2.7), $\varphi$ is given by Theorem 3.1 and

$$
\left\{\begin{aligned}
e= & \sum_{k=t}^{m-1} \min _{i, j \in I n} \mathcal{A}_{i, \alpha(k, j)}\left(\gamma^{\frac{k}{m}}-\gamma^{\frac{m-k}{m}}\right)+R-\sum_{k=t}^{m-1} \min _{i, j \in I n} \mathcal{A}_{i, \alpha(k, j)}, \\
f= & \sum_{k=t}^{m-1}\left(\begin{array}{c}
m-1 \\
m-k-1
\end{array}\right)(n-1)^{m-k-1} \beta_{m-k-1}(\mathcal{A})\left(\gamma^{\frac{k}{m}}-\gamma^{\frac{m-k}{m}}\right)+R \\
& -\sum_{k=t}^{m-1}\left(\begin{array}{c}
m-1 \\
m-k-1
\end{array}\right)(n-1)^{m-k-1} \beta_{m-k-1}(\mathcal{A}) \\
g= & r-\sum_{k=1}^{t-1} \min _{i, j \in I n} \mathcal{A}_{i, \alpha(k, j)}\left(1-\gamma^{-\frac{k}{m}}\right)-\sum_{k=t}^{m-1} \min _{i, j \in I n} \mathcal{A}_{i, \alpha(k, j)}, \\
h= & r-\sum_{k=1}^{t-1}\left(\begin{array}{c}
m-1 \\
m-k-1
\end{array}\right)(n-1)^{m-k-1} \beta_{m-k-1}(\mathcal{A})\left(1-\gamma^{-\frac{k}{m}}\right) \\
& \quad-\sum_{k=t}^{m-1}\left(\begin{array}{c}
m-1 \\
m-k-1
\end{array}\right)(n-1)^{m-k-1} \beta_{m-k-1}(\mathcal{A}) .
\end{aligned}\right.
$$

Obviously, we have

$$
\sum_{k=t}^{m-1}\left(\begin{array}{c}
m-1 \\
m-k-1
\end{array}\right)(n-1)^{m-k-1} \beta_{m-k-1}(\mathcal{A}) \geq \sum_{k=t}^{m-1} \min _{i, j \in I n} \mathcal{A}_{i, \alpha(k, j)} .
$$

Then $\left(1-\gamma^{-\frac{k}{m}}\right) \geq 0$, so it's easy to conclude that $h \leq g$. Since

$$
f-e=\sum_{k=t}^{m-1}\left(\left(\begin{array}{c}
m-1 \\
m-k-1
\end{array}\right)(n-1)^{m-k-1} \beta_{m-k-1}(\mathcal{A})-\min _{i, j \in I n} \mathcal{A}_{i, \alpha(k, j)}\right)\left(\gamma^{\frac{k}{m}}-\gamma^{\frac{m-k}{m}}-1\right),
$$

when $\gamma^{\frac{k}{m}}-\gamma^{\frac{m-k}{m}} \geq 1, k=t, \ldots, m-1$, we have $f \geq e$, i.e., $\varphi \geq \eta$.

So in some cases, the bound of Theorem 3.2 is sharper than that of (2.8).

Example 3.2 Let $\mathcal{A}=\left(a_{i_{1} i_{2} i_{3}}\right) \in \mathbb{R}_{+}^{[3,5]}$ with $k>4$,

$$
a_{111}=a_{1 i 1}=k, \quad a_{222}=a_{333}=a_{444}=a_{555}=1, \quad a_{i j j}=2,
$$

and other

$$
a_{i_{1} i_{2} i_{3}}=2 \text {. }
$$

Then $\mathcal{A}$ is a positive tensor, and we have $t=2$. A simple computation with (2.7) gives $\min _{i, j \in 5} \mathcal{A}_{i, \alpha(2, j)}=1, \min _{i, j \in 5} \mathcal{A}_{i, \alpha(1, j)}=4, R=5 k+40, r=49$, so

$$
\eta=\frac{\left[(\gamma)^{\frac{2}{3}}-(\gamma)^{\frac{1}{3}}\right]+5 k+40-1}{49-4\left(1-(\gamma)^{-\frac{1}{3}}\right)-1} .
$$

However, in Theorem 3.1, $\beta_{0}(\mathcal{A})=\min _{i, j} a_{i j \ldots j}=1, \beta_{1}(\mathcal{A})=2$, thus

$$
\varphi=\frac{\left[(\gamma)^{\frac{2}{3}}-(\gamma)^{\frac{1}{3}}\right]+5 k+40-1}{49-2 \times 4 \times 2\left(1-(\gamma)^{-\frac{1}{3}}\right)-1}=\frac{\left[(\gamma)^{\frac{2}{3}}-(\gamma)^{\frac{1}{3}}\right]+5 k+40-1}{49-16\left(1-(\gamma)^{-\frac{1}{3}}\right)-1} .
$$

Similarly, we take $k=10$,

$$
\eta=\frac{\left[(\gamma)^{\frac{2}{3}}-(\gamma)^{\frac{1}{3}}\right]+89}{44+4(\gamma)^{-\frac{1}{3}}}
$$




$$
\varphi=\frac{\left[(\gamma)^{\frac{2}{3}}-(\gamma)^{\frac{1}{3}}\right]+89}{32+16(\gamma)^{-\frac{1}{3}}} .
$$

So we have

$$
44+4(\gamma)^{-\frac{1}{3}}-32-16(\gamma)^{-\frac{1}{3}}=12\left(1-(\gamma)^{-\frac{1}{3}}\right)
$$

Since $\gamma \geq 1$, we get $\left(1-(\gamma)^{-\frac{1}{3}}\right) \geq 0$, and so

$$
\varphi \geq \eta .
$$

\section{Acknowledgements}

The authors would like to express their sincere thanks the anonymous referees and editors for their constructive comments and valuable suggestions.

\section{Funding}

This work was supported by the National Natural Science Foundation of P.R. China (Grant No. 11571095, 11601134, 11701154)

\section{Availability of data and materials}

All data generated or analyzed during this study are included in this published article.

\section{Competing interests}

The authors declare that they have no competing interests.

\section{Authors' contributions}

All authors contributed equally to this work. All authors read and approved the final manuscript.

\section{Publisher's Note}

Springer Nature remains neutral with regard to jurisdictional claims in published maps and institutional affiliations.

Received: 19 June 2019 Accepted: 16 October 2019 Published online: 24 October 2019

\section{References}

1. Bai, X., Huang, Z., Wang, Y.: Global uniqueness and solvability for tensor complementarity problems. J. Optim. Theory Appl. 170, 72-84 (2016)

2. Bermann, A., Plemmons, R.: Nonnegative Matrices in the Mathematical Sciences. Academic Press, New York (1979)

3. Chang, K., Pearson, K., Zhang, T.: Perron-Frobenius theorem for nonnegative tensors. Commun. Math. Sci. 6(2), 507-520 (2008)

4. Chang, K., Pearson, K., Zhang, T.: Some variational principles for Z-eigenvalues of nonnegative tensors. Linear Algebra Appl. 438, 4166-4182 (2013)

5. Chang, K., Zhang, T.: On the uniqueness and non-uniqueness of the Z-eigenvector for transition probability tensors. J. Math. Anal. Appl. 408, 525-540 (2013)

6. Che, M., Cichocki, A., Wei, Y.: Neural networks for computing best rank-one approximations of tensors and its applications. Neurocomputing 267, 114-133 (2017)

7. Che, M., Qi, L., Wei, Y.: Positive-definite tensors to nonlinear complementarity problems. J. Optim. Theory Appl. 168, 475-487 (2016)

8. Chen, H., Qi, L.: Positive definiteness and semi-definiteness of even order symmetric Cauchy tensors. J. Ind. Manag. Optim. 11(4), 1263-1274 (2015)

9. Gowda, M.S., Luo, Z., Qi, L., Xiu, N.: Z-tensors and complementarity problems (2016) arXiv:1510.07933v2

10. He, J.: Bounds for the largest eigenvalue of nonnegative tensors. J. Comput. Anal. Appl. 20(7), 1290-1301 (2016)

11. He, J., Huang, T.Z.: Upper bound for the largest Z-eigenvalue of positive tensors. Appl. Math. Lett. 38, 110-114 (2014)

12. Horn, R., Johnson, C.: Matrix Analysis. Cambridge University Press, Cambridge (1991)

13. Hu, S., Qi, L.: Convergence of a second order Markov chain. Appl. Math. Comput. 241, 183-192 (2014)

14. Hu, S., Qi, L., Zhang, G.: Computing the geometric measure of entanglement of multipartite pure states by means of non-negative tensors. Phys. Rev. A 93, 012304 (2016)

15. Huang, Z., Qi, L.: Formulating an $n$-person noncooperative game as a tensor complementarity problem. Comput. Optim. Appl. 66(3), 557-576 (2017)

16. Kempe, D., Kleinberg, J., Tardos, É.: Maximizing the spread of influence through a social network. Theory Comput. 11(4), 105-147 (2015)

17. Kofidis, E., Regalia, P.A.: On the best rank-1 approximation of higher-order supersymmetric tensors. SIAM J. Matrix Anal. Appl. 23, 863-884 (2002)

18. Li, L., Boulware, D.: High-order tensor decomposition for large-scale data analysis. In: 2015 IEEE International Congress on Big Data, New York, NY, pp. 665-668 (2015) 
19. Li, S., Li, C., Li, Y: A new bound for the spectral radius of nonnegative tensors. Journal of Inequalities and Applications. 88 (2017)

20. Li, W., Liu, D., Vong, S.W.: Z-eigenpair bounds for an irreducible nonnegative tensor. Linear Algebra Appl. 483 182-199 (2015)

21. Li, W., Liu, W., Vong, S.W.: Some bounds for H-eigenpairs and Z-eigenpairs of a tensor. J. Comput. Appl. Math. 342, 37-57 (2018)

22. Li, W., Ng, M.: On the limiting probability distribution of a transition probability tensor. Linear Multilinear Algebra 62, 362-385 (2014)

23. Lim, L.H.: Singular values and eigenvalues of tensors: a variational approach. In: Proceedings of the IEEE International Workship on Computational Advances in Multi-Sensor Adaptive Processing, CAMSAP 05, vol. 1, pp. 129-132. IEEE Computer Society Press, Piscataway (2005)

24. Ling, C., He, H., Qi, L.: On the cone eigenvalue complementarity problem for higher-order tensors. Comput. Optim. Appl. 63, 143-168 (2016)

25. Ling, C., He, H., Qi, L.: Higher-degree eigenvalue complementarity problems for tensors. Comput. Optim. Appl. 64(1), 149-176 (2016)

26. Luo, Z., Qi, L., Xiu, N.: The sparsest solutions to Z-tensor complementarity problems. Optim. Lett. 11(3), 471-482 (2017)

27. Ni, Q., Qi, L., Wang, F.: An eigenvalue method for testing positive definiteness of a multivariate form. IEEE Trans. Autom. Control 53, 1096-1107 (2008)

28. Qi, L.: Eigenvalues of a real supersymmetric tensor. J. Symb. Comput. 40, 1302-1324 (2005)

29. Qi, L.: Rank and eigenvalues of a supersymmetric tensor, the multivariate homogeneous polynomial and the algebraic hypersurface it defines. J. Symb. Comput. 41, 1309-1327 (2006)

30. Qi, L.: The best rank-one approximation ratio of a tensor space. SIAM J. Matrix Anal. Appl. 32(2), 430-442 (2011)

31. Qi, L.: Symmetric nonnegative tensors and copositive tensors. Linear Algebra Appl. 439, 228-238 (2013)

32. Qi, L., Chen, H., Chen, Y.: Tensor Eigenvalues and Their Applications. Springer, Singapore (2018)

33. Qi, L., Luo, Z: Tensor Analysis: Spectral Theory and Special Tensors. SIAM, Philadelphia (2017)

34. Song, Y., Qi, L.: Spectral properties of positively homogeneous operators induced by higher order tensors. SIAM J. Matrix Anal. Appl. 34, 1581-1595 (2013)

35. Song, Y., Qi, L.: Positive eigenvalue-eigenvector of nonlinear positive mappings. Front. Math. China 9(1), 181-199 (2014)

36. Song, Y., Qi, L.: Necessary and sufficient conditions for copositive tensors. Linear Multilinear Algebra 63(1), 120-131 (2015)

37. Song, Y., Qi, L.: Properties of some classes of structured tensors. J. Optim. Theory Appl. 165(3), 854-873 (2015)

38. Song, Y., Qi, L.: Tensor complementarity problem and semi-positive tensors. J. Optim. Theory Appl. 169, 1069-1078 (2016)

39. Song, Y., Qi, L.: Eigenvalue analysis of constrained minimization problem for homogeneous polynomial. J. Glob. Optim. 64(3), 563-575 (2016)

40. Song, Y., Yu, G.: Properties of solution set of tensor complementarity problem. J. Optim. Theory Appl. 170, 85-96 (2016)

41. Wang, Y., Huang, Z., Bai, X.: Exceptionally regular tensors and tensor complementarity problems. Optim. Methods Softw. 31, 815-828 (2016)

42. Will, C.: Theory and Experiment in Gravitational Physics. Cambridge University Press, Cambridge (1981)

43. Xie, J., Chang, A.: $\mathrm{H}$-Eigenvalues of signless Laplacian tensor for an even uniform hypergraph. Front. Math. China 8 107-128 (2013)

44. Xu, C.: Hankel tensors, Vandermonde tensors and their positivities. Linear Algebra Appl. 491, 56-72 (2016)

45. Yang, Q., Yang, Y.: Further results for Perron-Frobenius theorem for nonnegative tensors II. SIAM J. Matrix Anal. Appl. $32(4), 1236-1250(2011)$

46. Yang, Y., Yang, Q.: Further results for Perron-Frobenius theorem for nonnegative tensors. SIAM J. Matrix Anal. Appl. 31(5), 2517-2530 (2010)

47. Yang, Y., Yang, Q., Qi, L.: Properties and methods for finding the best rank-one approximation to higher-order tensors. Comput. Optim. Appl. 58, 105-132 (2014)

48. Zhang, M.: Social Data Analytics Using Tensors And Sparse Techniques. PhD Dissertations, The University of Texas at Arlington (2014)

49. Zhang, T., Golub, G.H.: Rank-one approximation to high order tensors. SIAM J. Matrix Anal. Appl. 23, 534-550 (2001)

\section{Submit your manuscript to a SpringerOpen ${ }^{\circ}$ journal and benefit from:}

- Convenient online submission

- Rigorous peer review

- Open access: articles freely available online

- High visibility within the field

- Retaining the copyright to your article

Submit your next manuscript at $\boldsymbol{~ s p r i n g e r o p e n . c o m ~}$ 